\title{
An assessment of early mandibular growth
}

EF. Hutchinson ${ }^{1,3}$, EN. L'Abbé ${ }^{1}, A C$. Oettlé ${ }^{2}$

1. Department of Anatomy, Section: Physical Anthropology, School of Medicine, Faculty of Health Sciences, University of Pretoria, South Africa

2. Department of Anatomy, Section: Clinical Anatomy, School of Medicine, Faculty of Health Sciences, University of Pretoria, South Africa.

3. School of Anatomical Sciences, Wits Medical School, Faculty of Health Sciences, University of the Witwatersrand

Total number of figures: $\quad 3$

Total number of tables: $\quad 4$

${ }^{*}$ E Hutchinson (Corresponding Author)

7 York Road

Parktown North,

Johannesburg

2193

E-Mail:erin.hutchinson@wits.ac.za

Tel: +2711 7172420 Fax: +2711 717242 


\begin{abstract}
Quantification of skeletal data has been shown to be an effective and reliable method of demonstrating variation in human growth as well as for monitoring and interpreting growth. In South Africa as well as internationally, few researchers have assessed mandibular growth in late fetal period and early childhood and therefore standards for growth and age determination in these groups are limited. The purpose of this study was to evaluate growth in the mandible from the period of 31 gestational weeks to 36 months postnatal. A total of 74 mandibles were used. Dried mandibles were sourced from the Raymond A. Dart Collection (University of Witwatersrand), and cadaveric remains were obtained from the Universities of Pretoria and the Witwatersrand. The sample was divided into four groups; 31 to 40 gestational weeks (group 1), 0 to 11 months (group 2), 12 to 24 months (group 3 ), and 25 to 36 months (group 4). Twenty-one osteological landmarks were digitized using a MicroScribe G2. Ten standard measurements were created and included: the maximum length of mandible, mandibular body length and width, mandibular notch width and depth, mental foramen to inferior border of mandible, mandibular basilar widths bigonial and biantegonial, bigonial width of mental foramen and mental angle. Data were analyzed using PAST statistical software and Morphologika2 v2.5. Statistically significant differences were noted in the linear measurements for all group comparisons except between groups 3 and 4 . The mandible morphologically changed from a round, smooth contour anteriorly to adopt a more sharp and narrow adult shape. A progressive increase in the depth and definition of the mandibular arch was also noted. In conclusion, the mandible initially grows to accommodate the developing tongue (up to 11 months), progressive dental eruption and mastication from 12 to 36 months. Mastication is associated with muscle mass development; this would necessitate an increase in the dimensions of the mandibular notch and associated muscle attachment sites. These findings might be valuable in the estimation of age in unidentified individuals and to monitor prenatal growth of the mandible for the early diagnosis of conditions associated with stunted mandibular growth.
\end{abstract}

Keyword: Geometric morphometrics, fetal, neonatal, mandible, growth 


\section{Introduction:}

Quantification of skeletal data has been shown to be an effective and reliable method of demonstrating variation in human growth as well as for monitoring and interpreting the growth of various skeletal elements in the living [1-3]. Despite numerous studies using osteometric techniques in the calculation of fetal and post-childhood mandibular growth, little research has focused on morphological changes - associated with growth and development - in the mandible between late term-fetuses and early childhood $[4,5]$.

The purpose of this study was firstly to document standard measurements on wet mandibular specimens and dried bone from 31 gestational weeks to 36 months postnatal and secondly to assess changes both osteometrically and morphologically between age categories including prenatal groups, from 31 to 40 gestational weeks, and postnatal groups from 0 to 11 months, 12 to 24 months and 25 to 36 months. From this data, a growth pattern could be discerned and interpreted. Knowledge of normal growth trends may be applied to more reliably diagnose abnormal changes in growth and development of the mandible. This osteometric and morphological information may also aid in the estimation of age of unidentified persons.

If undetected, abnormal mandibular growth in a fetus may present with life threatening complications at birth. Anomalies, which are associated with inadequate mandibular development, include Micrognathia, Hallermann-Streiff syndrome, Robinson anomalad and retrusion of the mandible and may occur as often as 1 in every 1000-recorded births in the USA [6]. If diagnosed early and delivery of the infant is successful, then treatment of these conditions may include an immediate tracheotomy and, in later teenage years, distraction osteogenesis (bone stretching) [7].

\section{Materials and methods:}

\section{Materials}

Both skeletal and cadaveric tissues were sourced from the Raymond A. Dart Collection and School of Anatomical Sciences at the University of Witwatersrand, South Africa, while only cadaveric remains were obtained from the Department of Anatomy, University of Pretoria, South Africa [8]. A total of 74 mandibles were used, the majority $(n=50)$ were obtained from the School of Anatomical Sciences at the University of Witwatersrand, while 24 were from the University of Pretoria.

As the age at death for the cadaver specimens was unknown, standard techniques for age estimation including crown-rump length, crown-heel length, toe-heel length and head circumference, were 
employed to determine an approximate gestational age reported in weeks and the various measurements in centimeters (Table 1) $[9,10,11]$. The age at death of the skeletal component $(n=22)$ on the other hand was estimated by using dental eruption and the morphological features of each mandible. This technique included observing which teeth had erupted as well as fusion of the mandibular symphysis. The skeletal elements were placed into an age category of $0,1,2$ and 3 years [12]. No fetal skeletal specimens were available and all skeletal samples used did indicate fusion of the symphysis menti.

Table 1. Sample composition and age distribution

\begin{tabular}{|c|c|c|c|}
\hline & \multicolumn{2}{|c|}{$\begin{array}{l}\text { School of Anatomical Sciences, } \\
\text { University of the Witwatersrand }\end{array}$} & \multirow{2}{*}{$\begin{array}{c}\text { Department of Anatomy, } \\
\text { University of Pretoria }\end{array}$} \\
\hline & $\begin{array}{l}\text { Cadaveric } \\
\text { Specimens }\end{array}$ & $\begin{array}{c}\text { Raymond Dart } \\
\text { Skeletal } \\
\text { Collection }\end{array}$ & \\
\hline $\begin{array}{l}\text { Group 1: } 31 \text { to } 40 \\
\text { gestational weeks }\end{array}$ & 18 & & \\
\hline $\begin{array}{l}\text { Group 2: } 0 \text { to } 11 \\
\text { months postnatal }\end{array}$ & 10 & 7 & 24 \\
\hline $\begin{array}{l}\text { Group 3: } 12 \text { to } 24 \\
\text { months postnatal }\end{array}$ & & 8 & \\
\hline $\begin{array}{l}\text { Group } 4: 25 \text { to } 36 \\
\text { months postnatal }\end{array}$ & & 7 & \\
\hline Total & 28 & 22 & 24 \\
\hline
\end{tabular}

\section{Methods}

\section{Dissection}

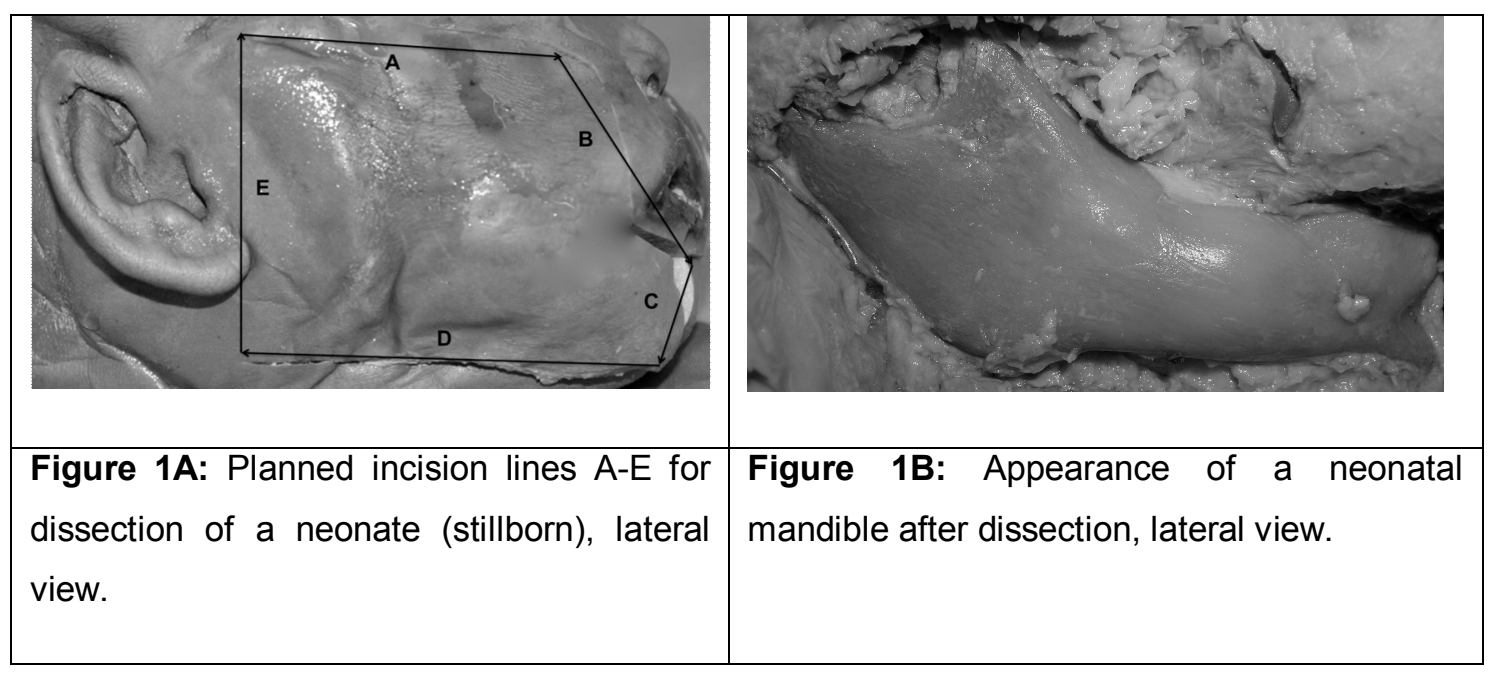


Figure 1: Planned incision lines for dissection of a neonate (stillborn), School of Anatomical Sciences, University of the Witwatersrand

Soft tissue dissections were performed using a standard dissection kit. Soft tissue was removed so as to expose the antero-lateral surfaces as well as the inferior border of the mandible. Dissection along the antero-lateral surface of the face included 5 incisions (Figure 1). The first incision, A was from the zygomatic process of the temporal bone, along the zygomatic arch towards the temporal process of the zygomatic bone. This was followed by incisions connecting the temporal process of the zygomatic bone and the superior border of the symphysis menti, $\mathrm{B}$, along the symphysis menti from the most superior to the most inferior points, $C$ and then from the inferior border of the symphysis menti to the mandibular angle (gonial angle), D. The dissections were then concluded with an incision connecting the posterior border of the mandibular angle to the zygomatic process of the temporal bone, E.

All musculature and neurovascular structures occurring on the antero-lateral surfaces of the mandible were reflected as one layer. This was done as a means to preserve the overlying soft tissue and to fully expose the bony landmarks for future research projects.

\section{Collection of landmark data}

An Immersion Microscribe $\mathrm{G} 2$ with an accuracy of $0.38 \mathrm{~mm}$ was used to record 21 landmarks on the mandible. These landmarks included both the points necessary to calculate the linear measurements and to define the shape of the mandible. The data was imported into Microsoft Excel ${ }^{\mathrm{TM}}$. Each mandible was positioned with the mandibular condyles inferiorly and the symphysis menti superiorly. Landmarks were recorded in a pre-set sequence, as illustrated in Figure 2.

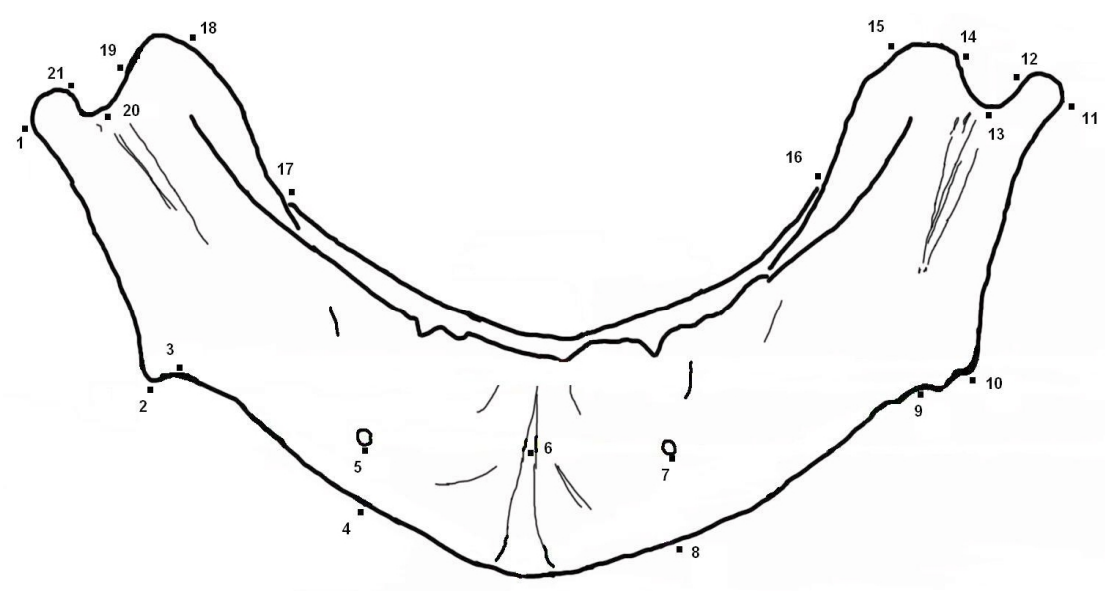


Figure 2: $\quad$ An illustration of the 21 homologous landmarks recorded on each mandible.

Table 2. A description of the homologous landmarks on the mandible

\begin{tabular}{|c|c|}
\hline Point & Description \\
\hline 1 & Most posterior point on the right condylar process \\
\hline 2 & Right gonial angle \\
\hline 3 & Deepest point on the Antegonial notch (right) \\
\hline 4 & Inferior border of mandible directly below the right mental foramen \\
\hline 5 & Mental foramen (right) \\
\hline 6 & $\begin{array}{l}\text { Symphysis menti / symphyseal ridge, on the same horizontal level as the mental } \\
\text { foramina }\end{array}$ \\
\hline 7 & Mental foramen (left) \\
\hline 8 & Inferior border of the mandible directly below the left mental foramen \\
\hline 9 & Deepest point on the Antegonial notch (left) \\
\hline 10 & Left gonial angle \\
\hline 11 & Most posterior point on the left condylar process \\
\hline 12 & Most anterior point on the left condylar process \\
\hline 13 & Deepest point on the mandibular notch on the left \\
\hline 14 & Most posterior point on the left coronoid process \\
\hline 15 & Most anterior point on the left coronoid process \\
\hline 16 & $\begin{array}{l}\text { Left oblique line termination/point on the oblique line where the body and ramus of } \\
\text { the mandible meet }\end{array}$ \\
\hline 17 & $\begin{array}{l}\text { Right oblique line termination/point on the oblique line where the body and ramus of } \\
\text { the mandible meet }\end{array}$ \\
\hline 18 & Most anterior point on the right coronoid process \\
\hline 19 & Most posterior point on the right coronoid process \\
\hline
\end{tabular}




\begin{tabular}{|l|l|}
\hline $\mathbf{2 0}$ & Deepest point on the mandibular notch on the right \\
\hline $\mathbf{2 1}$ & Most anterior point on the right condylar process \\
\hline
\end{tabular}

With each landmark, three coordinates $(\mathrm{X}, \mathrm{Y}, \mathrm{Z})$ were recorded and represent the horizontal, vertical and depth planes. The 3-dimensional co-ordinates were imported into a Microsoft Excel worksheet and the Pythagorean formula was used to convert the data into linear distances:

$$
=\sqrt{ }\left(x_{1}-x_{2}\right)^{2}+\left(y_{1}-y_{2}\right)^{2}+\left(z_{1}-z_{2}\right)^{2}
$$

where $x_{1}, y_{1}, z_{1}$ and $x_{2}, y_{2}, z_{2}$ refer to the coordinates of the first and second landmarks respectively.

\section{Osteometric analysis}

Nine standard anthropometric measurements as adapted from Hesby et al. (2006) and Scheuer \& Black (2000), were recorded, as well as the mental angle measurement, which was designed for the purpose of this study $[5,12,13]$.

\section{Mental angle (MA); Figure 2}

The angle formed at the mental symphysis between the mandibular rami of both sides of the mandible. The mental angle was calculated using the cosine rule and included values obtained from measurements 2 and 9 .

\section{Mandibular basilar width bigonial (MBS_BG) [5]; Figure 2 (point 2 to 10)}

The width of the mandibular arch measured from the bilateral angles of the mandible (point where the posterior and inferior borders meet).

\section{Mandibular basilar width bi-antegonial (MBS_BAG) [5]; Figure 2 (point 3 to 9)}

The width of the mandible measured from the bilateral points at the deepest point on the antegonial notch. 


\section{Bigonial width of mental foramen (BW_MF) [13]; Figure 2 (point 5 to7)}

Distance measured between the two mental foramina on the anterior surface of the mandible.

5. Mental foramen distance from inferior border of the mandible (MF_IBL/R) [13]; Figure 2 (point 5 to 4 and point 7 to 8 )

Distance measured from the mental foramen to the inferior border of the mandible directly below it.

6. Greatest depth of the mandibular notch (MND_L/R) [13]; Figure 2 (at point 13 and at point 20)

The line bisecting the width of the mandibular notch at its highest point to the deepest point of the mandibular notch.

7. Width of the mandibular notch (MNW_L/R) [13]; Figure 2 (point 12 to 14 and point 19 to 21)

The measurement taken from the inner surfaces of the mandibular condyle to the coronoid process.

8. Mandibular width (MW_L/R) [12]; Figure 2 (point 1 to 21 and point 11 to 15)

The measurement taken from the most posterior tip of the mandibular condyle to the most anterior tip of the coronoid process, i.e. the widest part of the ramus between the coronoid and condylar processes of the mandible

9. Mandibular body length (MBL_L/R) [12]; Figure 2 (point 2 to 6 and point 10 to 6 )

Distance measured from the mental tubercle of the mandible to the angle of the mandible. In cases where the two halves of the mandible had not fused the mental symphysis was then used as a reference point.

10. Longest length of the mandible (LLM_L/R) [12]; Figure 2 (point 1 to 6 and point 11 to 6 ) 
The distance from the mental tubercle to the posterior condylar tip of the mandible.

The cosine rule was used to calculate the mental angle from the mandibular body length of each side as well as the mandibular basilar width bigonial. The mandibular notch depth was calculated using the points from the mandibular notch width and greatest depth measurements and applied to the Pythagorean equation.

\section{Geometric morphometric analysis}

To analyze the landmarks in terms of shape, data was imported into Morphologika2 v2.5. A generalized full procrustes analysis was performed, which eliminated the influence of size as well as rotation and translation of the mandible. With a generalized full procrustes analysis, the landmarks were superimposed and compared with each other. In doing so, shape variation between the mandibles could be assessed. The reflections enabled option was selected, the function of this option is to superimpose landmark configurations of individual mandibles by minimizing the difference between each mandible.

In order to perform this analysis, the function required for translation, scaling and rotation, was enabled to remove the information not related to geometric shape. As procrustes superimposition renders the data inappropriate for the application of standard linear statistical methods the configurations needed to be projected orthogonally to the tangent space (see Rohlf, 1999 Journal of Classification 16:197-223). Principal component analysis was used to summarize the data for exploratory analysis (decomposes the data).

\section{Osteometric statistical analysis}

In order to assess the relationship between the linear measurements with independent variables such as age at death and sex, the data was evaluated in terms of distribution, i.e. normally distributed or skewed either to the left or to the right. A Shapiro-Wilk test was also conducted to assess for normality. Due to the left skewed nature of the data, a Kruskal-Wallis test was used to compare the various age groups [14]. Data was assessed at a $95 \%$ confidence interval. Ten specimens were evaluated by an independent observer and then re-evaluated by the primary investigator to determine inter-observer error. Values between 0.75 and 0.99 were considered indicative of a high degree of correlation (negative or positive values applied) [14]. 


\section{Results:}

\section{Osteometry:}

The size of the mandible from the prenatal ( 31 to 40 gestational weeks) and postnatal ( 0 to 11 months; 12 to 24 months; 25 to 36 months) groups was recorded so as to establish mean values for mandibular growth. This was achieved through the analysis of linear measurements and differences between the groups for the various age at death categories.

Descriptive statistics for each of the four groups is presented in Table 3. As expected, a general increase in size was observed for all osteometric dimensions from Groups 1 to 4 . As no statistically significant differences were noted between left and right, only the mean values for the left side are shown.

Table 3: Mean values ( $\mathrm{mm}$ ) and standard deviations for each of the measurements taken for groups $1-4$

\begin{tabular}{|c|c|c|c|c|c|c|c|c|}
\hline & \multicolumn{2}{|c|}{$\begin{array}{c}\text { Group } 1 \\
\text { 31-40 weeks }\end{array}$} & \multicolumn{2}{|c|}{$\begin{array}{c}\text { Group } 2 \\
\text { 0-11 months }\end{array}$} & \multicolumn{2}{|c|}{$\begin{array}{c}\text { Group } 3 \\
12-24 \text { months }\end{array}$} & \multicolumn{2}{|c|}{$\begin{array}{c}\text { Group } 4 \\
\text { 24-36 months }\end{array}$} \\
\hline \multirow[t]{2}{*}{$\mathbf{N}=$} & \multicolumn{2}{|c|}{18} & \multicolumn{2}{|c|}{41} & \multicolumn{2}{|c|}{8} & \multicolumn{2}{|c|}{7} \\
\hline & Mean & sd & Mean & sd & Mean & sd & Mean & sd \\
\hline MA & 58.16 & 16.66 & 68.56 & 8.06 & 69.03 & 5.89 & 72.18 & 4.05 \\
\hline MBS_BG & 32.64 & 7.24 & 41.41 & 7.86 & 53.32 & 5.26 & 61.14 & 6.00 \\
\hline MBS_BAG & 28.43 & 5.79 & 36.90 & 8.14 & 49.38 & 4.73 & 55.52 & 4.88 \\
\hline BW_MF & 21.09 & 6.32 & 24.90 & 6.09 & 32.51 & 2.78 & 34.19 & 2.58 \\
\hline MFIB_L & 6.38 & 1.48 & 6.13 & 2.34 & 7.02 & 1.21 & 6.74 & 0.71 \\
\hline MFIB_R & 5.86 & 0.92 & 6.31 & 2.16 & 6.91 & 1.47 & 7.42 & 1.36 \\
\hline MND_L & 4.21 & 1.23 & 3.47 & 1.65 & 6.77 & 1.77 & 7.70 & 2.28 \\
\hline MND_R & 3.60 & 0.94 & 3.39 & 1.53 & 6.35 & 1.69 & 7.29 & 2.78 \\
\hline MNW_L & 6.61 & 1.48 & 7.44 & 2.22 & 11.12 & 1.85 & 13.60 & 2.50 \\
\hline
\end{tabular}




\begin{tabular}{|c|c|c|c|c|c|c|c|c|}
\hline MNW_R & 7.52 & 2.67 & 7.92 & 2.33 & 11.91 & 1.75 & 14.49 & 3.02 \\
\hline MW_L & 15.38 & 1.98 & 16.91 & 3.59 & 20.86 & 2.27 & 23.90 & 2.98 \\
\hline MW_R & 17.00 & 5.45 & 16.48 & 3.69 & 21.55 & 2.27 & 24.83 & 2.51 \\
\hline MBL_L & 34.24 & 5.23 & 37.04 & 5.66 & 47.07 & 3.76 & 51.65 & 4.65 \\
\hline MBL_R & 30.56 & 4.20 & 36.46 & 5.94 & 47.23 & 3.83 & 52.21 & 5.16 \\
\hline LLM_L & 45.17 & 5.73 & 48.40 & 8.68 & 66.47 & 5.47 & 73.61 & 7.26 \\
\hline LLM_R & 41.85 & 6.26 & 47.66 & 9.23 & 67.37 & 5.52 & 74.15 & 6.88 \\
\hline
\end{tabular}

When the Kruskal-Wallis test was performed, statistically significant differences were noted in certain dimensions in some instances between the various groups, the values of which are presented in Table 4. As expected, a comparison between group 1 (31 to 40 gestational weeks) and group 4 (24 to 36 months) showed a statistically significant increase for all dimensions.

Table 4: Kruskall-wallis analysis, statistically significant differences in values for measurements taken when comparing groups 1-4

\begin{tabular}{|c|c|c|c|}
\hline & \multicolumn{3}{|c|}{ Groups } \\
\hline & $1-2$ & $2-3$ & 3-4 \\
\hline MA & 0.023 & & \\
\hline MBS_BG & 0.004 & 0.001 & \\
\hline MBS_BAG & 0.003 & 0.0016 & \\
\hline BW_MF & & 0.0058 & \\
\hline MND_L & & 0.00184 & \\
\hline MND_R & & 0.00225 & \\
\hline MNW_L & & 0.0034 & \\
\hline MNW_R & & 0.00183 & \\
\hline MW_L & & 0.03123 & \\
\hline MW_R & & 0.002269 & \\
\hline
\end{tabular}




\begin{tabular}{|c|c|c|l|}
\hline MBL_L & & 0.0008 & \\
\hline MBL_R & 0.00328 & 0.0003 & \\
\hline LLM_L & & 0.0003 & \\
\hline LLM_R & & 0.0001 & \\
\hline
\end{tabular}

Statistically significant increases in size were observed in the mental angle, the mandibular basilar widths bigonial and bi-antegonal, and the bigonial width of the mental foramen for most of the groups compared. An increase in these dimensions can be used to suggest that the mandible widens from the late fetal period to 3 years of age. This widening may be associated with the development and eruption of the decidious dentition as well as growth of the tongue.

An increase in the mental angle (MA) from age groups one to four is shown in table 3. In group one, the mean value for the mental angle was $58.16^{\circ}$, whereas in group 2, this increased to $68.56^{\circ}$. This increase of $10.4^{0}(18 \%)$ within the first year of life and was statistically significant $(p<0.05)$. After 12 months, the size of the mental angle did not substantially change and ranged from $69^{\circ}$ to $72^{\circ}$. The discrepancy between the first year of life and later years may be attributed to the fact that the mandibular symphysis usually fuses between 4 and 6 months, postnatal.

A statistically significant increase in size was illustrated in both the mandibular basilar widths bigonial and biantegonial, from the late fetal stage to 3 years of age. All differences between the groups were statistically significant, with the exception of groups 3 and 4 (12 to 36 months).

Differences in size for the bigonial width of the mental foramina (BW_MF) and the distance between the mental foramen and inferior border of the mandible (MF_IBL/R) from group 1 to 4 are presented in tables 3 and 4 . A statistically significant change with maturation was noted in the bigonial width of the mental foramina between group 2 ( 0 to 11 months) with $24.90 \mathrm{~mm}$ and group 3 ( 12 to 24 months) with $32.51 \mathrm{~mm}$. Therefore from the first year of life to the second the posterior mandible notably widens and this may be attributed to the development of the tongue.

Statistically significant increases in size were only observed between birth and 2 years of age for the mandibular notch depth and width, the length of the mandibular body, and the longest length of the mandible (tables 3 and 4). The mandibular notch depth (tables 3 and 4) showed a statistically significant difference $(p<0.001)$ between groups $2(0-11$ months $)(3.47 \mathrm{~mm})$ and $3(12-24$ months $)$ 
$(6.77 \mathrm{~mm})$. This increase, between 12 and 24 months of age, may be attributed to the development and eruption of the decidious dentition as well as an increased reliance on mastication during this period. This correlates well with the previously observed increases in the arch dimensions to accommodate other masticatory structures e.g. the developing tongue.

When the measurements were assessed for the level of repeatability a range indicating values between 0.79 and 0.99 was specified. This indicated a high level of measurement repeatability both for inter-observer as well as intra-observer purposes. The distance between the inferior border of the mandible and the mental foramen was the least repeatable with a value of 0.78 and a range of between 0.41 and 0.93 . This may be attributed to difficulties in locating the exact point of the inferior border of the mandible.

\section{Geometric morphometrics}

Morphological changes associated with maturity were assessed using geometric morphometrics. Changes in mandibular shape were expressed in terms of their distribution along principle component axes 1 and 2, where the most variation was indicated (Figure 3). Principle component 1 (PC1) accounted for the majority of variance found in the sample (scores range from -0.36 to +0.32 ) as indicated by $86 \%$. PC2 only minimally influenced the variance in the sample (scores range from -0.08 to +0.08 ) as indicated by $14 \%$.

In both PC1 and PC2, the general trend is that there is a high degree of overlap between groups 1 and 2 as well as between groups 3 and 4 (Figure 3). This is consistent with observations made osteometrically, where no statistically significant changes were observed between consecutive age groups, with the exception of groups 2 (0 to 11 months) and 3 (12 to 24 months). The statistically significant difference observed between groups 2 and 3 are further shown in the large degree of separation between these groups along the PC1 and PC2 axes.

The following changes were noted on either the thin-plate splines or the wireframes in the specimens along the PC1 and 2 axes. The posterior region of the mandible indicated an increase in the mandibular notch depth and width; this was morphologically evident by an increase in the definition of the mandibular notch area. There was also an increase observed in the rameal width of the mandible from groups 1 to 4 . A lateral protrusion (flaring) of the mandibular ramus region, particularly in the area of the gonion corresponded well with increases observed in the mandibular basilar widths bigonial and biantegonial, as both these measurements were taken using this area. As a result, the flaring observed also influenced the increase indicated in the mental angle 
On the anterior aspect of the mandible a change in the shape of the mandibular arch and mental region from a smooth and round shape to a more sharp and narrow appearance gave the developing mandible a slightly more adult shape. The increased morphological definition in the mental region (through bone replacing the symphyseal cartilage) also increased the distance between the laterally located mental foramina and as such increased the bigonial width of the mental foramina.

Subsequently when morphological changes in the posterior and anterior regions are further considered, they also increase the longest length of the mandible and the mandibular body length measurements. The shape of the mandibular arch in changing from a short round to a longer narrow arch shape anteriorly with a posterior bulging, led to the posteriorly placed condylar processes being located further from the central axis. This in turn increased the longest length of the mandible measurement. 


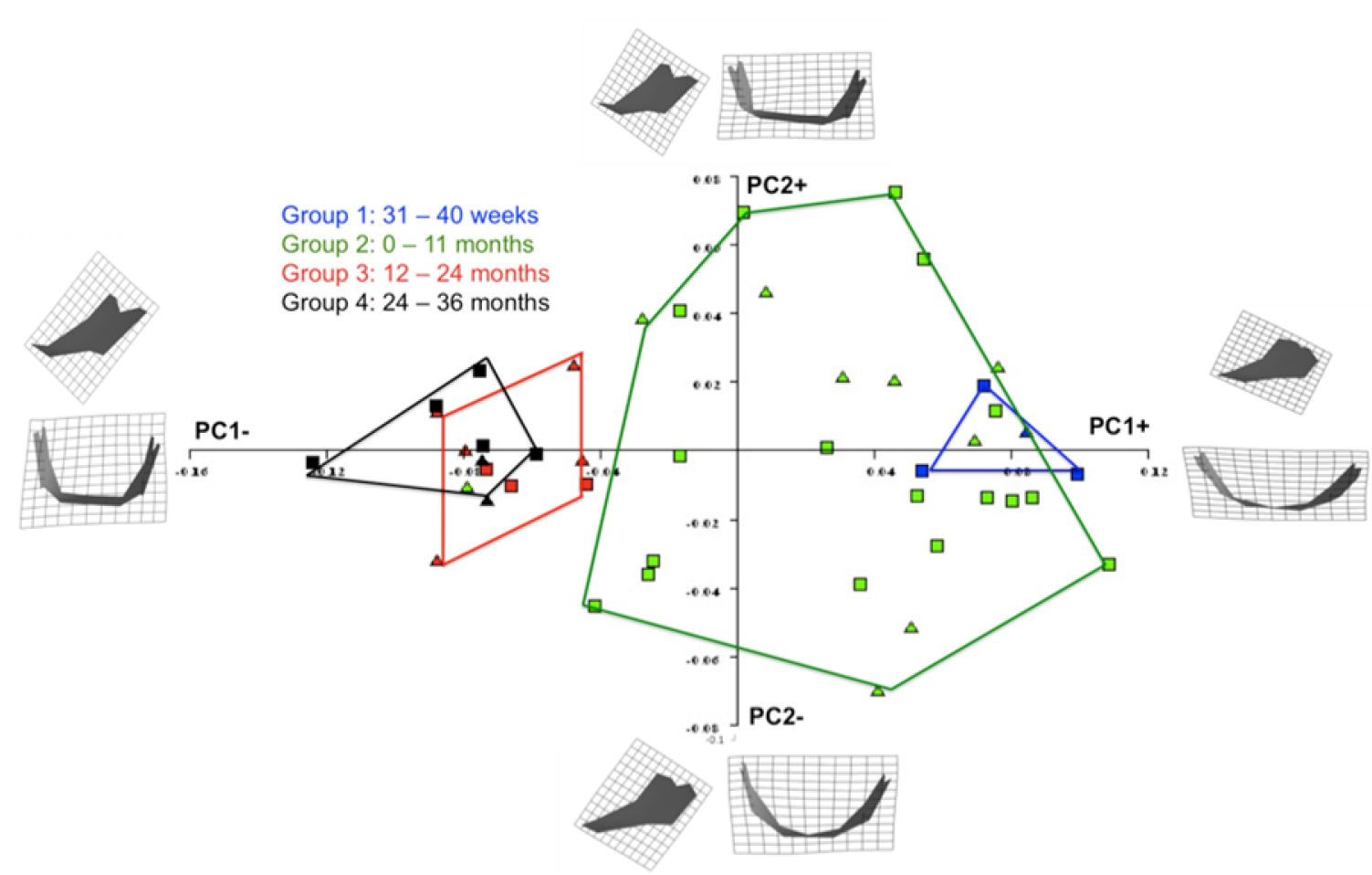

Figure 3: $\quad$ Distribution of variance along principle components 1 and 2 comparing groups 1-4 


\section{Summary}

In general, the increase in mandibular size and change in shape were statistically insignificant from the late fetal period (31 to 40 gestational weeks) to 11 months. However when comparing the first year of life to the second, the mandible was observed to widen significantly posteriorly (increases in the bigonial and biantegonial widths) and lengthen (increase in the body length and longest length of the mandible) in preparation and accommodation of the dentition.

\section{Discussion}

In South Africa, no osteometric standards exist in which changes in mandibular growth from the late fetal period to three years of age can be evaluated. For clinicians, this is a crucial period for the diagnosis and treatment of conditions such as micrognathia or Hallermann-Streiff syndrome. Additionally, dumped fetal and neonatal remains are frequently discovered in a fragmentary condition. An anthropologist is often requested to provide an assessment of age at death and sex from these remains. Thus the purpose of this study was to document changes in the size and shape of the mandible and to compare it with age at death.

From 31 gestational weeks to 36 months, a general increase in size was noted in the mandible, which included: a lengthening of the body, a widening of the basilar arch and a flaring of the ramus. These changes may be attributed to growth and development of the tongue, fusion of the symphysis menti, development and eruption of the deciduous dentition, and influence from the associated muscles of mastication.

An increase in the mandibular body length and a posterior widening of the bigonial and biantegonial dimensions from the late fetal through the first year of life may primarily be associated with development and growth of the tongue as well as the development of the deciduous dentition. In a study on prenatal growth of the mandible from 5 to 40 gestational weeks, Lee (2001) noted that the tongue commenced its growth in an anterior direction during the $5^{\text {th }}$ gestational week $[15,16]$. In the 6th gestational week, the genioglossus muscle (extrinsic tongue muscle) attached to the posterior surface of the mandibular symphysis [16]. This attachment to the posterior surface, the author suggests, may have resulted in the tongue movements being responsible for the early induction of mandibular movement and growth [16]. Lee (2001) further stated that as masticatory and tongue muscles from the 8th gestational week support the mandible, it would be able to control the development of the lower jaw even in the absence of Meckel's cartilage [16]. Thus growth and development of the tongue through associated musculature attachment would have had an influence 
on the increase in the size and changes in shape of the arch dimensions analyzed in this study; especially in terms of the anterior (mental foramen width) and posterior (bigonial width) dimensions.

The influence of the developing dentition on the changes in the mandibular arch and body length dimensions may be attributed to the interaction between the mesenchymal cells and dental epithelium. Fleichmannova et al. (2010) describes how the mesenchymal cells associated with the oral cavity differentiate into dentin producing odontoblasts, when contact is made with the dental epithelium [17]. The superficial mesenchymal part of the tooth germ ultimately gives rise to structures including the alveolar bone [17]. The author also suggests that intramembranous ossification is triggered in regions where the mesenchymal cells are subject to a shearing detraction that originates from growth of tissues at varying speeds $[17,18]$. In addition to allow for proper alignment of the mandibular dentition, the tooth size must be in harmony with the arch size $[17,18]$. As a result the increase noted in the mandibular body length and arch dimensions may be attributed to the signals arising from the dental follicle, which regulate bone reabsorption and deposition during phases of tooth development and eruption.

During the transition between the pre-natal and birth periods, alterations in the mandibular arch dimensions were noted. Continual growth of the tongue may have been an influencing factor in the increase in the mandibular arch. Further increases in the longest length of the mandible, mandibular width, bigonial width of the mental foramen and mandibular notch dimensions were also observed. Increases in the mandibular basilar widths - bigonial and biantegonial - indicate a posterior widening of the mandible with maturity. As in the fetal period an increase in the mandibular body length with maturation after birth can be attributed to continual growth of the tongue as well as development and eruption of the dentition. Eruption of the deciduous dentition would be observable from 6 to 36 months. Scheuer and Black (2000) suggest that the morphology of the developing mandible is attributed to bone deposition occurring posteriorly and bone resorption anteriorly [12]. The resultant effect is the posterior displacement of the ramus and an increase in mandibular body length so as to accommodate the erupting dentition. This was observed in the current study with the increase in the mandibular body length. The deciduous central incisors erupt around 6 months of age. The lateral incisors follow at 10 months, first molars at 14 months, canines at 17 months and the second molars erupting between 23 and 24 months, depending on the sex [12].

Changes in mandibular body length (MBL) are emphasized through a change in orientation of the mandibular ramus to the mandibular body. With maturation, the mandibular ramus becomes more upright in position and this may be the result of the deposition of bone on the superior aspect of the mandibular body along with a progression in dental eruption [12]. 
Dental eruption also accounts for changes expressed in the mandibular angle. Scheuer and Black (2000) noted that the mandibular angle decreased from $150^{\circ}$ at birth to $130^{\circ}$ after the eruption of the deciduous teeth [12]. The angle of the mandible may have influenced the longest length of the mandible. A change in mandibular angulation is most evident when comparing mandibles from different age groups. While at birth, the angle of the mandible is reported to be obtuse (closer to $180^{\circ}$ ), during growth the angle becomes more acute (closer to $90^{\circ}$ ). These changes observed in the mandibular angle indicate a possible relationship with the longest length of the mandible. As the angle of the mandible became more acute, it decreased the distance between the most posterior point of the mandibular condyle and the most anterior point of the symphysis menti. Therefore, the previously observed increase in the body length coupled with the changes in the mandibular angle may have also influenced changes in the longest length of the mandible.

The longest length of the mandible may also be influenced by the position of the mandibular condyles, as well as the width of the ramus and notch. Both of these dimensions were shown to have statistically significant increases, postnatally. These increases along with increases in the mandibular notch depth may also have been due to an increase use in the muscles of mastication.

Furthermore as the sample consisted of both cadaveric and skeletonized specimens, shrinkage of tissues might be considered to influence the mental angle measurements in those specimens with an unfused mandible. The significant difference seen in the mental angle is found between groups 1 and 2 which are of mixed composition i.e. group 1 comprises cadaveric specimens where group 2 comprises both cadaveric and skeletonized specimens. The significant difference indicated in this comparison may be indicative of tissue changes however due to the change being found in the transition from cadaveric to skeletonized specimens, the expected result would be a decrease in the angle and not a significant increase as was found. The increase in the mental angle although not significant was found to persist throughout the entire assessment and thus indicated a general increase in the angle which may be associated with an increase in the general arch dimensions.

\section{Conclusion:}

In general the mandible changed to accommodate three main events. The first change noted was between the prenatal and early postnatal groups (31 gestational weeks to 11 months postnatal). The increase in the mandibular dimensions noted during this period was suggested to occur as a result of the mandible growing in synchronization with the developing tongue and deciduous dentition. The second major change noted was between 12 and 24 months postnatal, where the mandible adapted its dimensions to accommodate the eruption of the deciduous dentition. The third change was across 
the entire postnatal group ( 0 to 36 months), following dental eruption and increase in mastication. The increase in mastication had a two-fold effect on changes in the size and shape of the mandible. Firstly, an increase in the muscle mass would necessitate an increase in the dimensions of the mandibular notch [19]. Secondly, an increase in use of these muscles imposed an increase in stress and strain on the muscle attachment sites resulting in a more pronounced morphology in these areas. These findings especially that of the osteometric dimensions of this study are envisaged to be valuable in the estimation of age in unidentified individuals and to monitor prenatal growth of the mandible so as to facilitate the early diagnosis of conditions associated with stunted mandibular growth.

\section{Tables and Figures:}

Figures:

Figure 1: $\quad$ Planned incision lines for dissection of a neonate (stillborn), School of Anatomical Sciences, University of the Witwatersrand

Figure 2: $\quad$ An illustration of the 21 homologous landmarks recorded on each mandible.

Figure 3: $\quad$ Distribution of variance along principle components 1 and 2 comparing groups 1-4

\section{Tables:}

Table 1: $\quad$ Sample composition and age distribution

Table 2. A description of the homologous landmarks on the mandible

Table 3: $\quad$ Mean values $(\mathrm{mm})$ and standard deviations for each of the measurements taken for groups 1-4

Table 4: Kruskall-wallis analysis, statistically significant differences in values for measurements taken when comparing groups 1-4

\section{References:}

1. White TD, Folkens PA. The human bone manual. Amsterdam (Netherlands): Elsevier Academic Press; 2005; p. 340-1.

2. Oettlé AC, Pretorius E, Steyn M. Geomorphometric analysis of mandibular ramus flexure. Am J Phys Anthropol 2005;128:623-9.

3. Oettlé AC, Pretorius E, Steyn M. Geometric morphometric analysis of the use of mandibular gonial eversion in sex determination. HOMO 2009;60:29 - 43. 
4. Fazekas \& Kosa. Forensic fetal osteology. Budapest (Hungary): Akademiai Kiado; 1978; $p$. 171-196.

5. Hesby RM, Marshall SD, Dawson DV, Southard KA, Casko JS, Franciscus RG, et al. Transverse skeletal and dentoalveolar changes during growth. Am J Orthodontics \& Dentofacial Orthopedics 2006; December 1;6:721-31.

6. Giangluigi P, Kypros N, Renato X, Philippe J. Diploma in fetal medicine [Online]. 2002 [cited 2008 June 3]; Available from URL:http://www.centrus.com.br/DiplomaFMF/SeriesFMF/18-23weeks/index-18.htm

7. Lansford M. Advances in neonatal care. 2008; 8(6):308-14 [online]. [cited 2008 Aug]; Available from: URL: http://www.advancesinneonatalcare/org/pt/re/anc/

8. Krogman WM, Iscan MY. The human skeleton in forensic medicine. $2^{\text {nd }}$ ed. Springfield (IL): CC Thomas; 1986.

9. Lubchenco LO, Hansman C, Boyd E. Intrauterine growth in length and head circumference as estimated from live births at gestational ages from 26 to 42 weeks. Pediatrics. 1966; 37:403-8 [online]. [cited 2008 March]; Available from URL: http://www.pediatrics.org

10. Hansen K, Sung CJ, Huang C, Pinar H, Singer DB, Oyer CE. Reference values for second trimester fetal and neonatal organ weights and measurements. J. Pediatrics and developmental pathology. 2003; 6:160-7

11. Fenton TR. A new growth chart for preterm babies: Babson and Brenda's chart updated with recent data and a new format. BMC Pediatrics. 2003; 3(13): 1-10

12. Scheuer L, Black S, editors. The juvenile skeleton. San Diego (CA): Elsevier Academic Press; 2000 .

13. De Villiers $\mathrm{H}$. The skull of the South African negro: a biometrical and morphological study. Johannesburg (RSA): Witwatersrand University Press; 1968.

14. Allan JC. Learning about statistics: a primer in simple statistical methods for students of the medical, biological, paramedical, social and behavioral sciences. Johannesburg (RSA): Macmillan South Africa (Pty)(Ltd); 1982.

15. Carlson BM. Human embryology and developmental biology. $3^{\text {rd }}$ ed. Philadelphia (PA): Mosby Inc; 2004. p. 317-351.

16. Lee SK, Kim YS, Oh HS, Yang KH, Kim EC, Chi JG. Prenatal development of the human mandible. Anat rec 2001; 263: 314-325.

17. Fleischmannova J, Matalova E, Sharpe PT, Misek I, Radlanski RJ. Formation of the toothbone interface. J Dental Research 2010; 89(2): 108-115 
18. Moorrees CFA, Reed RB. Biometrics of crowding and spacing of teeth in the mandible. Amer. J. Phys. Anthrop 1954; 12: 77-88

19. Berkovitz BKB, Holland GR, Moxham BJ. Oral anatomy, histology and embryology. $4^{\text {th }}$ ed. Edinburgh (Scotland): Mosby Elsevier; 2009. 